\title{
SOME INTEGRAL FORMULAE ON WEIGHTED MANIFOLDS
}

\author{
MOHAMMED ABDELMALEK AND MOHAMMED BENALILI
}

\begin{abstract}
Introducing a notion of the weighted mean $\sigma_{r}^{\infty}$-curvature and using the weighted Newton transformations we derive in this paper some integral formulae on weighted manifolds. These formulae generalize the flux formula and some of its examples of applications obtained by Alias, de Lira and Malacarne 3 .
\end{abstract}

\section{INTRODUCTION}

Several works have been done in the past to study the geometric properties of constant $k$-mean curvature hypersurfaces in space forms, where the $k$-mean curvature is defined as the $k$-th elementary symmetric function of the eigenvalues of the second fundamental form see ([3]). Motivated in part by connections with the Ricci flow, much works have also been done on geometric properties of manifolds and hypersurfaces when the manifold is endowed with a "weighted" volume element; i.e. one integrates using the smooth measure $e^{-f} d v o l_{g}$ for $d v o l_{g}$ the Riemannian volume element of the metric $g$ see ( [6], [7], 8], [9], [13] ). In this work, we follow Case ([6]) to introduce the notion of weighted $k$-mean curvature and using the weighted Newtons transformations introduced in ([6]) we obtain an integral formula on weighted manifolds and give some applications. This latter formula was first introduced by Kusner in ([12]) and nowadays it's called the flux formula. Then it was extended to $k$-curvature in a nice paper by Alías, de Lira, and Malacarne see ([3]). Where they studied the properties of certain geometrical configurations, more particularly they established a flux formula and gave examples of geometric applications. Our paper extends some properties obtained by the authors cited above for weighted manifolds.

1991 Mathematics Subject Classification. Primary 53C21, 5342.

Key words and phrases. weighted manifolds, weighted $\sigma_{r}$-mean curvatures, weighted Newtons transformations. 


\section{Preliminaries}

In this section we fix the notations and recall some definitions and properties of the weighted symmetric functions and the weighted Newton transformations: for more details see $([6],[13])$.

Given a complete $n$-dimensional Riemannian manifold $(M,\langle\rangle$,$) and a$ smooth function $f: M \longrightarrow \mathbb{R}$. The weighted manifold $M_{f}$ associate to $M$ is the triplet $\left(M,\langle\rangle,, d v_{f}\right)$, where $d v_{f}=e^{-f} d v$ and $d v$ is the standard volume element of $M$.

Consider the tensional connection

$$
\widetilde{\nabla}_{X} Y=\nabla_{X} Y+\langle X, Y\rangle V-\langle V, Y\rangle X
$$

where $V=\langle\nabla f, \nu\rangle \nu, \nu$ is a vector field on $M$ orthogonal to $X$ and $\nabla$ stands for the covariant derivative on $M$. This connection is one of the three basic types of metric connections introduced by Elie Cartan. It was studied by I. Agricola and M. Kraus [2].

If $B$ and $\widetilde{B}$ are second forms on $M$ defined by $B(X, Y)=\left\langle\nabla_{X} Y, \nu\right\rangle$ and $\widetilde{B}(X, Y)=\left\langle\widetilde{\nabla}_{X} Y, \nu\right\rangle$, we have

$$
\widetilde{B}(X, Y)=B(X, Y)+\langle X, Y\rangle\langle\nabla f, \nu\rangle
$$

An example of this situation is as follows: let $\psi_{t}: M^{n} \rightarrow\left(\bar{M}^{n+1},\langle\rangle,\right)$ be a one family of parameter of immersions of an $n$-dimensional manifold $M^{n}$ into an $n+1-\bar{M}^{n+1}$ Riemannian manifold where $\nabla$ is the connection induced on $M^{n}$ and $\widetilde{\nabla}$ is the the tonsorial connection on $M^{n}$, so the mean curvatures of $M$ with respective to the connections $\nabla$ and $\widetilde{\nabla}$ are related by

$$
n \widetilde{H}=n H+\langle\nabla f, \nu\rangle
$$

which is the classical weighted mean curvature.

The important point is that hypersurfaces of constant weighted curvature appear as critical points of certain weighted volume functionals. The fundamental analogy with constant mean curvature hypersurfaces draws the attention in this area. In terms of matrices we have

$$
\widetilde{B}=B+\langle\nabla f, \nu\rangle I
$$

so if $\widetilde{\tau}_{i}$ and $\tau_{i}$ are the eigenvalues of $\widetilde{B}$ and $B$ respectively, we get

$$
\widetilde{\tau}_{i}=\tau_{i}+\langle\nabla f, \nu\rangle .
$$

Now, putting $\lambda=\langle\nabla f, \nu\rangle$, we obtain [1]

$$
\widetilde{\sigma}_{k}=\sigma_{k}(\widetilde{B})=\sum_{j=0}^{k}\left(\begin{array}{c}
n-k+j \\
j
\end{array}\right) \lambda^{j} \sigma_{k-j}(B) .
$$


where $\widetilde{\sigma}_{k}$ stand for the symmetric functions of $\widetilde{B}$.

Let $\bar{M}$ be an $(n+1)$-dimensional Riemannian manifold, and $\psi$ : $M \longrightarrow \bar{M}$ be an isometrically immersed hypersurface with $\nabla$ and $\bar{\nabla}$ the Levi-Civita connections on $M$ and $\bar{M}$ respectively. The Weingarten formula of this immersion is written as follows

$$
A X=-\left(\bar{\nabla}_{X} N\right)^{\top}
$$

where $A$ is the shape operator of the hypersurface $M$ with respect to the Gauss map $N$, and ${ }^{\top}$ denotes the orthogonal projection on the tangent vector bundle of $M$. As it is well known $A$ is a linear self adjoint operator and at each point $p \in M$, its eigenvalues $\mu_{1}, \ldots, \mu_{n}$ are the principal curvatures of $M$.

Associate to the shape operator $A$ are the weighted elementary symmetric functions $\sigma_{k}^{\infty}: \mathbb{R} \times \mathbb{R}^{n} \longrightarrow \mathbb{R}$ defined recursively ( and introduced by Case in [6] ) by

$$
\left\{\begin{array}{l}
\sigma_{0}^{\infty}\left(\mu_{0}, \mu\right)=1, \\
k \sigma_{k}^{\infty}\left(\mu_{0}, \mu\right)=\sigma_{k-1}^{\infty}\left(\mu_{0}, \mu\right) \sum_{j=0}^{n} \mu_{j}+\sum_{i=1}^{k-1} \sum_{j=1}^{n}(-1)^{i} \sigma_{k-1-i}^{\infty}\left(\mu_{0}, \mu\right) \mu_{j}^{i} \quad \text { for } k \geq 1
\end{array}\right.
$$

where $\mu_{0} \in \mathbb{R}$ and $\mu=\left(\mu_{1}, \ldots, \mu_{n}\right) \in \mathbb{R}^{n}$. In particular for $\mu_{0}=0$ we recover $\sigma_{k}^{\infty}(0, \mu)=\sigma_{k}(\mu)$ the classical elementary symmetric functions defined in [14].

Definition 1. ([6])The weighted Newton transformations (W.N.T) $T_{k}^{\infty}\left(\mu_{0}, A\right)$ are defined inductively from $A$ by :

$$
\left\{\begin{array}{l}
T_{k}^{\infty}\left(\mu_{0}, A\right)=I \\
T_{k}^{\infty}\left(\mu_{0}, A\right)=\sigma_{k}^{\infty}\left(\mu_{0}, A\right) I-A T_{k-1}^{\infty}\left(\mu_{0}, A\right) \quad \text { for } k \geq 1
\end{array}\right.
$$

or equivalently

$$
T_{k}^{\infty}\left(\mu_{0}, A\right)=\sum_{j=0}^{k}(-1)^{j} \sigma_{k-j}^{\infty}\left(\mu_{0}, A\right) A^{j}
$$

where I stands for the identity on the Lie algebra of vector fields $\varkappa(M)$, $\sigma_{k}^{\infty}\left(\mu_{0}, A\right)=\sigma_{k}^{\infty}\left(\mu_{0}, \mu_{1}, \ldots, \mu_{n}\right)$ and $\mu_{1}, \ldots, \mu_{n}$ are the eigenvalues of $A$.

It should be noted that $T_{k}^{\infty}(0, A)=T_{k}(A)$ is the classical Newton transformations introduced in [14].

These functions enjoy the nice following properties.

Proposition 1. 6] For $\mu_{0}, \mu_{1} \in \mathbb{R}$ and $\mu \in \mathbb{R}^{n}$, we have

$$
\sigma_{k}^{\infty}\left(\mu_{0}+\mu_{1}, \mu\right)=\sum_{j=0}^{k} \frac{\mu_{1}^{j}}{j !} \sigma_{k-j}^{\infty}\left(\mu_{0}, \mu\right) .
$$


In particular,

$$
\begin{gathered}
\sigma_{k}^{\infty}\left(\mu_{1}, \mu\right)=\sum_{j=0}^{k} \frac{\mu_{1}^{j}}{j !} \sigma_{k-j}(\mu) \\
\operatorname{trace}\left(A T_{k}^{\infty}\left(\left(\mu_{0}, \mu\right)\right)\right)=(k+1) \sigma_{k+1}^{\infty}\left(\mu_{0}, \mu\right)-\mu_{0} \sigma_{k}^{\infty}\left(\mu_{0}, \mu\right) .
\end{gathered}
$$

For $i \in\{1, \ldots, n\}$ we have

$$
\sigma_{k, i}^{\infty}\left(\mu_{0}, \mu\right)=\sigma_{k}^{\infty}\left(\mu_{0}, \mu\right)-\mu_{i} \sigma_{k-1, i}^{\infty}\left(\mu_{0}, \mu\right)
$$

and the $i^{\text {th }}$ eigenvalue of $T_{k}^{\infty}\left(\mu_{0}, \mu\right)$ is equal to $\sigma_{k, i}^{\infty}\left(\mu_{0}, \mu\right)$ where $\sigma_{k, i}^{\infty}\left(\mu_{0}, \mu\right)=$ $\sigma_{k}^{\infty}\left(\mu_{0}, \mu_{1}, \ldots, \mu_{i-1}, \mu_{i+1}, \ldots, \mu_{n}\right)$.

We can see by (2.1) and (2.2) that $\widetilde{\sigma}_{k}$ and $\sigma_{k}^{\infty}\left(\mu_{0}, \mu\right)$ are polynomials of the same degree but with slightly different coefficients, which differ by a multiplicative constant only.

Definition 2. The weighted $k^{\text {th }}$ mean curvature $H_{k, f}$ is given by:

$$
\left(\begin{array}{c}
n \\
k
\end{array}\right) H_{k, f}=\sigma_{k}^{\infty}(\langle\nu, \nabla f\rangle, A)
$$

where $\nu$ is the unit outpointing vector field normal to $M$ in $\bar{M}$.

Remark 1. In particular for $k=1$ and in view of formula 2.2, we get

$$
n H_{1, f}=\sigma_{1}^{\infty}(\langle\nu, \nabla f\rangle, A)=\sigma_{1}(A)+\langle\nu, \nabla f\rangle=n H+\langle\nu, \nabla f\rangle
$$

which is the classical definition of the weighted mean curvature of the hypersurface $M$ studied by Gromov [10].

To clarify this notion of curvature we will study the case $k=2$.

Consider a one family of parameter $\psi_{t}: M^{n} \rightarrow \bar{M}^{n+1}(c)$ of immersions of an $n$-dimensional closed manifold $M^{n}$ into an $n+1$ space form $\left(\bar{M}^{n+1},\langle\rangle,\right)$ of constant curvature $c$. Denote by $X$ the deformation vector field and by $\nu$ the normal vector field to $\bar{M}^{n+1}$. Put $\lambda=\langle X, \nu\rangle$.

Consider the variational problem

$$
\delta\left(\int_{M} \sigma_{1}^{\infty} d V_{f}\right)=0
$$


that is to say

$$
\begin{aligned}
(2 . \delta)\left(\int_{M} \sigma_{1}^{\infty} d V_{f}\right) & =\frac{d}{d t}\left(\int_{M} \sigma_{1}^{\infty} d V_{f}\right) \\
& =\frac{d}{d t}\left(\int_{M}\left(\sigma_{1}+\mu\right) d V_{f}\right) \\
& =\int_{M}\left(\frac{d \sigma_{1}}{d t}+\frac{d \mu}{d t}\right) d V_{f}+\int_{M}\left(\sigma_{1}+\mu\right) \frac{d\left(d V_{f}\right)}{d t} .
\end{aligned}
$$

Now, by formula (9c) in page 469, we have

$$
\frac{d \sigma_{1}}{d t}=\lambda\left(\sigma_{1}^{2}-2 \sigma_{2}\right)+\lambda_{, i i}+\sigma_{1, j} X^{j}+n c \lambda
$$

and by the well known fact $\frac{d}{d t} d V=\left(-\lambda \sigma_{1}+X_{, j}^{j}\right) d V$, we infer

$$
\frac{d\left(d V_{f}\right)}{d t}=\left(-\lambda \sigma_{1}-X^{j} f_{, j}+X_{, j}^{j}\right) d V_{f}
$$

and

$$
\frac{d \mu}{d t}=\left\langle\nabla \nabla_{X} f, \nu\right\rangle-\langle\nabla f,[\nu, X]\rangle
$$

By the definition of the weighted divergence, we have $d i v_{f} X=-X^{j} f_{, j}+$ $X_{, j}^{j}$ and replacing in (2.5), we get

$$
\begin{aligned}
\frac{d}{d t}\left(\int_{M} \sigma_{1}^{\infty} d V_{f}\right)= & \int_{M}\left(\lambda\left(\sigma_{1}^{2}-2 \sigma_{2}\right)+\lambda_{, i i}+\sigma_{1, j} X^{j}++n c \lambda+\left\langle\nabla \nabla_{X} f, \nu\right\rangle-\langle\nabla f,[\nu, X]\rangle\right) d V \\
& +\int_{M}\left(\sigma_{1}+\mu\right)\left(-\lambda \sigma_{1}+d i v_{f}(X)\right) d V_{f} \\
= & \int_{M}\left(-2 \lambda \sigma_{2}+\lambda_{, i i}+\left\langle\nabla \nabla_{X} f, \nu\right\rangle-\langle\nabla f,[\nu, X]\rangle\right) d V_{f} \\
& +\int_{M}\left(-\lambda \mu \sigma_{1}+\mu d i v_{f}(X)+n c \lambda\right) d V_{f} \\
= & \int_{M}\left(-\lambda\left(2 \sigma_{2}^{\infty}-\mu_{1} \sigma_{1}^{\infty}\right)+\lambda_{, i i}+\left\langle\nabla \nabla_{X} f, \nu\right\rangle-\langle\nabla f,[\nu, X]\rangle\right) d V_{f} \\
& +\int_{M}\left(\mu d i v_{f}(X)+n c \lambda\right) d V_{f}
\end{aligned}
$$

we have

Theorem 1. The Euler-Lagrange equation corresponding to the problem 2.4 is

$$
-\lambda\left(2 \sigma_{2}^{\infty}-\mu_{1} \sigma_{1}^{\infty}\right)+\lambda_{, i i}+\left\langle\nabla \nabla_{X} f, \nu\right\rangle-\langle\nabla f,[\nu, X]\rangle+\mu d i v_{f}(X)+n c \lambda=0 .
$$


To clarify the idea we will consider simpler cases: put $f(x)=\frac{1}{2}\|x\|^{2}$, and $X=g \nu$, where $g$ is a $C^{2}$-function, so $\lambda=g$ and $\mu=\langle x, \nu\rangle$ the support function. We get

$$
\begin{aligned}
\operatorname{div}_{f}(X) & =-\left(g \nu^{j}\right) f_{, j}+\left(g \nu^{j}\right)_{, j} \\
& =-g\langle x, \nu\rangle+g_{j} \nu^{j}+g \nu_{, j}^{j} \\
& =-g \mu+\nu(g)-g \sigma_{1} \\
& =g \sigma_{1}^{\infty}+\nu(g), \\
\int_{M} g_{, i i} d V_{f} & =\int_{M} g\left(f_{, i i}-f_{i} f^{, i}\right) d V_{f} \\
& =\int_{M} g(n-2 f) d V_{f}
\end{aligned}
$$

and

$$
\left\langle\nabla \nabla_{X} f, \nu\right\rangle-\langle\nabla f,[\nu, X]\rangle=g \nu\left(\mu_{1}\right)
$$

Hence

$$
\begin{aligned}
\frac{d}{d t}\left(\int_{M} \sigma_{1}^{\infty} d V_{f}\right)= & \int_{M}\left(-g\left(2 \sigma_{2}^{\infty}-2 \mu \sigma_{1}^{\infty}\right)+g(n-2 f)+g \nu(\mu)\right) d V_{f} \\
& +\int_{M} \mu \nu(g) d V_{f}
\end{aligned}
$$

and since

$$
\begin{aligned}
\int_{M} \mu \nu(g) d V_{f} & =-\int_{M} \nu\left(\mu e^{-f}\right) g d V \\
& =-\int_{M} g\left(\nu(\mu)-\mu^{2}\right) d V_{f}
\end{aligned}
$$

we deduce

$$
\frac{d}{d t}\left(\int_{M} \sigma_{1}^{\infty} d V_{f}\right)=\int_{M} g\left[-2 \sigma_{2}^{\infty}+2 \mu \sigma_{1}^{\infty}-2 f+\mu^{2}+n(1+c)\right] d V_{f} .
$$

Corollary 1. Under the above assumptions the Euler-Langrange equation of the problem (2.4) is given by

$$
-2 \sigma_{2}^{\infty}+2 \mu \sigma_{1}^{\infty}-2 f+\mu^{2}+n(1+c)=0 .
$$

Example: Consider a hypersurface $M_{n}$ of the unit sphere $S^{n+1}$. The Euler-Lagrange is then written

$$
-\sigma_{2}^{\infty}+\sigma_{1}^{\infty}+n=0
$$


or equivalently

$$
\left(2-\sum_{j=0}^{n} \mu_{j}\right) \sigma_{1}^{\infty}+2 n+\sum_{j=0}^{n} \mu_{j}=0
$$

where $\mu_{j}$ stand for the eigenvalues of the second fundamental form.

Or

$$
\left(\sigma_{1}^{\infty}\right)^{2}-4 \sigma_{1}^{\infty}-2 n-1=0
$$

which gives

$$
\sigma_{1}^{\infty}=2 \pm \sqrt{2 n+3}
$$

or

$$
\sigma_{1}=1 \pm \sqrt{2 n+3}
$$

We can cite a candidate to our situation: Cliffor torus in $S^{n+1}$ i.e. $M^{n}$ is a product of spheres $S^{n_{1}}\left(r_{1}\right) \times S^{n_{2}}\left(r_{2}\right), n_{1}+n_{2}=n$ of appropriate radii $r_{1}, r_{2}$. An $H(r)$-torus in $S^{n+1}$ is obtained by the canonical immersions $S^{n-1}(r) \subset R^{n}, S^{1}\left(\sqrt{1-r^{2}}\right) \subset R^{2}, 0<r<1$, as $S^{n-1}(r) \times S^{1}\left(\sqrt{1-r^{2}}\right) \subset S^{n+1}$. The principal curvatures are given, for a chosen orientation, by

$$
\mu_{1}=\ldots=\mu_{n-1}=\frac{\sqrt{1-r^{2}}}{r}, \mu_{n}=-\frac{r}{\sqrt{1-r^{2}}}
$$

so for the $H(r)$-torus

$$
\sigma_{1}=(n-1) \frac{\sqrt{1-r^{2}}}{r}-\frac{r}{\sqrt{1-r^{2}}}=\frac{n\left(1-r^{2}\right)-1}{r \sqrt{1-r^{2}}} .
$$

For the torus to be an example we have to show that

$$
\frac{n\left(1-r^{2}\right)-1}{r \sqrt{1-r^{2}}}=1+\sqrt{2 n+3}
$$

has a root. For this, we consider the continuous function $\phi(r)=-(1+$ $\sqrt{2 n+3}$ ) with $0<r<\frac{n-1}{n}$ and $n \geq 2$. We notice that $\lim _{r \rightarrow 0^{+}} \phi(r)=$ $+\infty$ and $\phi\left(\frac{n-1}{n}\right)=\frac{n}{\sqrt{2 n+1}}-1-\sqrt{2 n+3}<0$. Consequently the equation (??) admits at least one root.

Definition 3. We say that an hypersurface $M$ of $\bar{M}$ is $\sigma_{r}^{\infty}$-minimal, if $H_{r, f}=0$. In particular $M$ is $f$-minimal if $H_{f}=-\frac{1}{n}\langle\nu, \nabla f\rangle$.

Here we need $\mu_{0}=\langle\nabla f, \nu\rangle$. For the safe of brevity, we put $T_{k}^{\infty}=$ $T_{k}^{\infty}\left(\mu_{0}, A\right)$ and $\sigma_{k}^{\infty}=\sigma_{k}^{\infty}\left(\mu_{0}, A\right)$.

The weighted divergence of the weighted Newton transformations is define by

$$
\operatorname{div}_{f} T_{k}^{\infty}=e^{f} \operatorname{div}\left(e^{-f} T_{k}^{\infty}\right)
$$


where

$$
\operatorname{div}\left(T_{k}^{\infty}\right)=\operatorname{trace}\left(\nabla T_{k}^{\infty}\right)=\sum_{j=0}^{k} \nabla_{e_{i}}\left(T_{k}^{\infty}\right)\left(e_{i}\right)
$$

and $\left\{e_{1}, \ldots, e_{n}\right\}$ is an orthonormal basis of the tangent space of $M$.

\section{Lemma 1.}

$$
\operatorname{tr}\left(T_{k-1}^{\infty} \circ \nabla_{v} A\right)=\left\langle\nabla \sigma_{k}^{\infty}\left(\mu_{1}, A\right)-\sigma_{k-1}^{\infty}\left(\mu_{1}, A\right) \nabla \mu_{1}, v\right\rangle .
$$

Proof. The computations will be in a basis that diagonalizes $A$. Let

$$
\begin{aligned}
& A=\left(\begin{array}{ccc}
\lambda_{1} & & \\
& \cdot & \\
& & \lambda_{n}
\end{array}\right) \text {. Since the eigenvalues of } T_{k-1}^{\infty} \text { are given by } \\
& t_{i}=\sigma_{k-1}^{\infty}\left(\mu_{1}, \lambda_{1}, \ldots, \lambda_{i-1}, \lambda_{i+1}, \ldots, \lambda_{n}\right) \\
& =\sum_{j=0}^{k-1} \frac{\mu_{1}^{j}}{j !} \sigma_{k-1-j}\left(\lambda_{1}, \ldots, \lambda_{i-1}, \lambda_{i+1}, \ldots, \lambda_{n}\right) \\
& =\sum_{j=0}^{k-1} \frac{\mu_{1}^{j}}{j !} \frac{\partial}{\partial \lambda_{i}} \sigma_{k-1-j}\left(\lambda_{1}, \ldots, \lambda_{n}\right) \\
& =\sum_{j=0}^{k-1} \frac{\mu_{1}^{j}}{j !} \sum_{i \neq i_{j}, i_{1}<\ldots<i_{k-1-j}} \lambda_{i_{1}} \ldots \lambda_{i_{k-1-j}} .
\end{aligned}
$$

So

$$
\begin{aligned}
& \operatorname{tr}\left(T_{k-1}^{\infty} \circ \nabla_{v} A\right)=\sum_{i=1}^{n} t_{i} \nabla_{v} \lambda_{i} \\
= & \sum_{j=0}^{k} \frac{\mu_{1}^{j}}{j !} \nabla_{v} \sum_{i_{1}<\ldots<i_{k-j}} \lambda_{i_{1}} \ldots \lambda_{i_{k-j}} \\
= & \nabla_{v} \sigma_{k}^{\infty}\left(\mu_{1}, A\right)-\nabla_{v} \mu_{1} \sum_{j=0}^{k-1} \frac{\mu_{1}^{j}}{j !} \sigma_{k-1-j}(A) \\
= & \nabla_{v} \sigma_{k}^{\infty}\left(\mu_{1}, A\right)-\left(\nabla_{v} \mu_{1}\right) \sigma_{k-1}^{\infty}\left(\mu_{1}, A\right) .
\end{aligned}
$$

Lemma 2. The weighted divergence of the weighted Newton transformations $T_{k}^{\infty}$ are inductively given by the following formula

$$
\operatorname{div}_{f} T_{0}^{\infty}=\nabla f
$$


and

$\operatorname{div}_{f} T_{k}^{\infty}=\sigma_{k}^{\infty} \nabla f+\sigma_{k-1}^{\infty}\left(\mu_{1}, A\right) \nabla \mu_{1}-A \operatorname{div}_{f} T_{k-1}^{\infty}-\sum_{i=1}^{n}\left(\bar{R}\left(N, T_{k-1}^{\infty}\left(e_{i}\right)\right) e_{i}\right)^{\top} \quad$ for $k \geq 1$

Proof. We have

$$
\begin{aligned}
\operatorname{div}_{f} T_{k}^{\infty} & =e^{f} \operatorname{div}\left(e^{-f} T_{k}^{\infty}\right) \\
& =e^{f} \sum_{i=1}^{n}\left[\nabla_{e_{i}}\left(e^{-f} T_{k}^{\infty}\right)\left(e_{i}\right)\right] \\
& =e^{f} \sum_{i=1}^{n}\left[\nabla_{e_{i}}\left(e^{-f} T_{k}^{\infty}\left(e_{i}\right)\right)-e^{-f} T_{k}^{\infty}\left(\nabla_{e_{i}} e_{i}\right)\right] \\
& =e^{f} \sum_{i=1}^{n}\left[e^{-f} \nabla_{e_{i}}\left(T_{k}^{\infty}\left(e_{i}\right)\right)+e_{i}\left(e^{-f}\right) T_{k}^{\infty}\left(e_{i}\right)-e^{-f} T_{k}^{\infty}\left(\nabla_{e_{i}} e_{i}\right)\right] \\
& =e^{f} \sum_{i=1}^{n}\left[e^{-f} \nabla_{e_{i}}\left(T_{k}^{\infty}\left(e_{i}\right)\right)-e^{-f}\left\langle\nabla f, e_{i}\right\rangle T_{k}^{\infty}\left(e_{i}\right)-e^{-f} T_{k}^{\infty}\left(\nabla_{e_{i}} e_{i}\right)\right] \\
& =\operatorname{div} T_{k}^{\infty}-T_{k}^{\infty}(\nabla f)
\end{aligned}
$$

It is not difficult to see that

$$
\operatorname{div}_{M} T_{k}^{\infty}=\nabla \sigma_{k}^{\infty}-A_{k} \operatorname{div}_{M} T_{k-1}^{\infty}-\sum_{i=1}^{n}\left(\nabla_{e_{i}} A_{k}\right)\left(T_{k-1}^{\infty}\left(e_{i}\right)\right)
$$

Indeed,

$$
\begin{aligned}
\left(\nabla_{e_{i}} T_{k}^{\infty}\right)\left(e_{i}\right) & =\nabla_{e_{i}}\left(T_{k}^{\infty}\left(e_{i}\right)\right)-T_{k}^{\infty}\left(\nabla_{e_{i}} e_{i}\right) \\
& =\nabla_{e_{i}}\left(\left(\sigma_{k}^{\infty} I-A T_{k-1}^{\infty}\right) e_{i}\right)-\left(\sigma_{k}^{\infty} I-A T_{k-1}^{\infty}\right)\left(\nabla_{e_{i}} e_{i}\right) \\
& =\nabla_{e_{i}}\left(\sigma_{k}^{\infty} e_{i}\right)-\nabla_{e_{i}}\left(A T_{k-1}^{\infty}\left(e_{i}\right)\right)-\sigma_{k}^{\infty}\left(\nabla_{e_{i}} e_{i}\right)+\left(A T_{k-1}^{\infty}\right)\left(\nabla_{e_{i}} e_{i}\right) \\
& =e_{i}\left(\sigma_{k}^{\infty}\right) e_{i}+\sigma_{k}^{\infty}\left(\nabla_{e_{i}} e_{i}\right)-\nabla_{e_{i}}\left(A T_{k-1}^{\infty}\left(e_{i}\right)\right)-\sigma_{k}^{\infty}\left(\nabla_{e_{i}} e_{i}\right)+\left(A T_{k-1}^{\infty}\right)\left(\nabla_{e_{i}} e_{i}\right) \\
& =e_{i}\left(\sigma_{k}^{\infty}\right) e_{i}-\left(\nabla_{e_{i}}\left(A T_{k-1}^{\infty}\left(e_{i}\right)\right)-\left(A T_{k-1}^{\infty}\right)\left(\nabla_{e_{i}} e_{i}\right)\right) \\
& =\left(d_{e_{i}} \sigma_{k}^{\infty}\right)\left(e_{i}\right)-\left(\nabla_{e_{i}}\left(A T_{k-1}^{\infty}\right)\right) e_{i} \\
& =\left\langle\nabla \sigma_{k}^{\infty}, e_{i}\right\rangle e_{i}-\left(\nabla_{e_{i}} A\right)\left(T_{k-1}^{\infty}\left(e_{i}\right)\right)-A\left(\left(\nabla_{e_{i}} T_{k-1}^{\infty}\right)\left(e_{i}\right)\right)
\end{aligned}
$$


Thus,

$$
\begin{aligned}
\operatorname{div}_{M} T_{k}^{\infty} & =\sum_{i=1}^{n}\left(\nabla_{e_{i}} T_{k}^{\infty}\right)\left(e_{i}\right) \\
& =\sum_{i=1}^{n}\left\langle\nabla \sigma_{k}^{\infty}, e_{i}\right\rangle e_{i}-\sum_{i=1}^{n}\left(\nabla_{e_{i}} A\right)\left(T_{k-1}^{\infty}\left(e_{i}\right)\right)-\sum_{i=1}^{n} A\left(\left(\nabla_{e_{i}} T_{k-1}^{\infty}\right)\left(e_{i}\right)\right) \\
& =\nabla \sigma_{k}^{\infty}-\operatorname{Adiv}_{M} T_{k-1}^{\infty}-\sum_{i=1}^{n}\left(\nabla_{e_{i}} A\right)\left(T_{k-1}^{\infty}\left(e_{i}\right)\right)
\end{aligned}
$$

The Godazzi equation and the fact that $\nabla_{e_{i}} A$ is a self-ajoint operator allow us to write,

$\sum_{i=1}^{n}\left\langle\left(\nabla_{e_{i}} A\right) T_{k-1}^{\infty}\left(e_{i}\right), v\right\rangle=\left\langle\sum_{i=1}^{n}\left(\bar{R}\left(N, T_{k-1}^{\infty}\left(e_{i}\right)\right) e_{i}\right)^{\top}, v\right\rangle+\operatorname{trace}\left(T_{k-1}^{\infty} \circ \nabla_{v} A\right)$

where $v$ is an arbitrary vector tangent $M$.

Thus,

$\left\langle\operatorname{div}_{M} T_{k}^{\infty}, v\right\rangle=\left\langle\nabla \sigma_{k}^{\infty}, v\right\rangle-\left\langle\operatorname{Adiv}_{M} T_{k-1}^{\infty}, v\right\rangle-\left\langle\sum_{i=1}^{n}\left(\bar{R}\left(N, T_{k-1}^{\infty}\left(e_{i}\right)\right) e_{i}\right)^{\top}, v\right\rangle-\operatorname{tr}\left(T_{k-1}^{\infty} \circ \nabla_{v} A\right)$

Using now Lemma 1

$$
\operatorname{tr}\left(T_{k-1}^{\infty} \circ \nabla_{v} A\right)=\left\langle\nabla \sigma_{k}^{\infty}\left(\mu_{1}, A\right)-\left(\nabla \mu_{1}\right) \sigma_{k-1}^{\infty}\left(\mu_{1}, A\right), v\right\rangle
$$

we have,

$$
\left\langle\operatorname{div}_{M} T_{k}^{\infty}, v\right\rangle=\sigma_{k-1}^{\infty}\left(\mu_{1}, A\right)\left\langle\nabla \mu_{1}, v\right\rangle-\left\langle\operatorname{Adiv}_{M} T_{k-1}^{\infty}, v\right\rangle-\left\langle\sum_{i=1}^{n}\left(\bar{R}\left(N, T_{k-1}^{\infty}\left(e_{i}\right)\right) e_{i}\right)^{\top}, v\right\rangle
$$

or equivalently,

$$
\operatorname{div}_{M} T_{k}^{\infty}=\sigma_{k-1}^{\infty}\left(\mu_{1}, A\right) \nabla \mu_{1}-\operatorname{Adiv}_{M} T_{k-1}^{\infty}-\sum_{i=1}^{n}\left(\bar{R}\left(N, T_{k-1}^{\infty}\left(e_{i}\right)\right) e_{i}\right)^{\top}
$$


Finally

$$
\begin{aligned}
\operatorname{div}_{f} T_{k}^{\infty} & =T_{k}^{\infty}(\nabla f)+\sigma_{k-1}^{\infty}\left(\mu_{1}, A\right) \nabla \mu_{1}-\operatorname{Adiv}_{M} T_{k-1}^{\infty}-\sum_{i=1}^{n}\left(\bar{R}\left(N, T_{k-1}^{\infty}\left(e_{i}\right)\right) e_{i}\right)^{\top} \\
& =\left(\sigma_{k}^{\infty} I-A T_{k-1}^{\infty}\right)(\nabla f)+\sigma_{k-1}^{\infty}\left(\mu_{1}, A\right) \nabla \mu_{1}-A\left(\operatorname{div}_{f} T_{k-1}^{\infty}-T_{k-1}^{\infty}(\nabla f)\right)-\sum_{i=1}^{n}(\bar{R}(N \\
& =\sigma_{k}^{\infty} \nabla f+\sigma_{k-1}^{\infty}\left(\mu_{1}, A\right) \nabla \mu_{1}-\left(A T_{k-1}^{\infty}\right)(\nabla f)-A\left(\operatorname{div}_{f} T_{k-1}^{\infty}\right)+\left(A T_{k-1}^{\infty}\right)(\nabla f)-\sum_{i=1}^{n} \\
& =\sigma_{k}^{\infty} \nabla f+\sigma_{k-1}^{\infty}\left(\mu_{1}, A\right) \nabla \mu_{1}-A \operatorname{div}_{f} T_{k-1}^{\infty}-\sum_{i=1}^{n}\left(\bar{R}\left(N, T_{k-1}^{\infty}\left(e_{i}\right)\right) e_{i}\right)^{\top}
\end{aligned}
$$

Which achieves the proof of Lemma 2 .

Corollary 2. If $\bar{M}$ has constant sectional curvature, then

$$
\operatorname{div}_{f} T_{k}^{\infty}=T_{k}^{\infty}(\nabla f)+\sigma_{k-1}^{\infty}\left(\mu_{1}, A\right) \nabla \mu_{1} .
$$

Proof. If $\bar{M}$ has constant sectional curvature, then $\left(\bar{R}\left(N, T_{k-1}^{\infty}\left(e_{i}\right)\right) e_{i}\right)^{\top}=$ 0 , and we have

$$
\operatorname{div}_{f} T_{k}^{\infty}=\sigma_{k}^{\infty} \nabla f-A \operatorname{div}_{f} T_{k-1}^{\infty}+\sigma_{k-1}^{\infty}\left(\mu_{1}, A\right) \nabla \mu_{1} .
$$

The desired relation results by a recursive argument.

\section{MAin RESUlts}

The aim of this part is to derive an integral formula on weighted manifolds with constant sectional curvature and to give some of its geometric applications. The method is based on the computation of the weighted divergence $\operatorname{div}_{f}\left(T_{k}^{\infty} Y^{\top}\right)$ and $\left\langle\operatorname{div}_{f} T_{k}^{\infty}, Y\right\rangle$, where $Y$ is a conformal vector field. To do so, we first consider the following geometric configuration: let $\bar{M}^{n+1}$ be an oriented Riemannian manifold with metric $\langle\rangle,, P^{n} \subset \bar{M}^{n+1}$ an oriented connected submanifold of $\bar{M}^{n+1}$ and $\Sigma^{n-1} \subset P^{n}$ a compact hypersurface of $P^{n}$. Let $\varphi: M^{n} \longrightarrow$ $\bar{M}^{n+1}$ be a compact oriented hypersurface of boundary $\partial M$.

Let $p \in \Sigma^{n-1}$ and $\left\{e_{1}, \ldots, e_{n-1}\right\}$ an orthonormal basis of $T_{p} \Sigma^{n-1}$. We can choose a global vector field $\nu$ such that $\left\{e_{1}, \ldots, e_{n-1}, \nu(p)\right\}$ is an orthonormal basis of $T_{p} M^{n}$. Let $N$ be the globally vector normal to $M^{n}$, then $\left\{e_{1}, \ldots, e_{n-1}, \nu(p), N\right\}$ is an orthonormal basis of $T_{p} \bar{M}^{n+1}$.

Suppose now the existence of a closed conformal vector field $Y$ on $\bar{M}^{n+1}$; that is to say there exists a $\phi \in C^{\infty}\left(\bar{M}^{n+1}\right)$ such that

$$
\bar{\nabla}_{V} Y=\phi V
$$


for every vector fields $V$ over $\bar{M}^{n+1}$.

If $\left\{e_{1}, \ldots, e_{n}\right\}$ is an orthonormal basis of $T_{p} M^{n}$ that diagonalizes $A$, then

$$
\begin{aligned}
\left\langle\operatorname{div}_{f} T_{k}^{\infty}, Y\right\rangle & =\left\langle e^{f} \operatorname{div}\left(e^{-f} T_{k}^{\infty}\right), Y\right\rangle \\
& =e^{f} \operatorname{div}\left(e^{-f} T_{k}^{\infty} Y\right)-\sum_{i=0}^{n}\left\langle T_{k}^{\infty}\left(e_{i}\right), \nabla_{e_{i}} Y\right\rangle \\
& =e^{f} \operatorname{div}\left(e^{-f} T_{k}^{\infty} Y\right)-\sum_{i=0}^{n} \phi\left\langle T_{k}^{\infty}\left(e_{i}\right), e_{i}\right\rangle \\
& =\operatorname{div}_{f}\left(T_{k}^{\infty} Y\right)-\phi t r T_{k}^{\infty} .
\end{aligned}
$$

And in virtue of formula 2.3 we have

$$
\begin{aligned}
\operatorname{tr} T_{k}^{\infty} & =n \sigma_{k}^{\infty}\left(\mu_{0}, \mu\right)-\operatorname{tr}\left(A T_{k-1}^{\infty}\left(\mu_{0}, \mu\right)\right) \\
& =(n-k) \sigma_{k}^{\infty}\left(\mu_{0}, \mu\right)+\mu_{0} \sigma_{k-1}^{\infty}\left(\mu_{0}, \mu\right) \\
& =(n-k)\left(\begin{array}{c}
n \\
k
\end{array}\right) H_{k, f}+\langle\nu, \nabla f\rangle\left(\begin{array}{c}
n \\
k-1
\end{array}\right) H_{k-1, f} .
\end{aligned}
$$

So,

$$
\operatorname{div}_{f}\left(T_{k}^{\infty} Y\right)=\left\langle\operatorname{div}_{f} T_{k}^{\infty}, Y\right\rangle+\phi\left(c_{k} H_{k, f}+c_{k-1}\langle\nu, \nabla f\rangle H_{k-1, f}\right)
$$

where $c_{k}=(n-k)\left(\begin{array}{l}n \\ k\end{array}\right)$ and $c_{k-1}=n\left(\begin{array}{c}n \\ k-1\end{array}\right)$

Integrating the two sides of this latter equality and applying the divergence theorem, we obtain for $1 \leq k \leq n-1$,

$$
\begin{aligned}
\int_{M^{n}} \operatorname{div}_{f}\left(T_{k}^{\infty} Y\right) d v_{f} & =\int_{M^{n}} e^{-f} \operatorname{div}_{f}\left(T_{k}^{\infty} Y\right) d v \\
& =\int_{M^{n}} \operatorname{div}\left(e^{-f} T_{k}^{\infty} Y\right) d v \\
& =\int_{\partial M} e^{-f}\left\langle T_{k}^{\infty} Y, \nu\right\rangle d s \\
& =\int_{\partial M}\left\langle T_{k}^{\infty} Y, \nu\right\rangle d s_{f}
\end{aligned}
$$

Hence,

$\int_{\partial M}\left\langle T_{k}^{\infty} \nu, Y\right\rangle d s_{f}=\int_{M^{n}}\left\langle\operatorname{div}_{f} T_{k}^{\infty}, Y\right\rangle d v_{f}+c_{k} \int_{M^{n}} \phi H_{k, f} d v_{f}+c_{k-1} \int_{M^{n}} \phi\langle\nu, \nabla f\rangle H_{k-1, f} d v_{f}$.

Consequently, we have the following proposition 
Proposition 2. Let $\varphi: M^{n} \longrightarrow \bar{M}^{n+1}$ an immersed compact oriented hypersurface of boundary $\partial M$. Denoting by $N$ the global vector fields normal to $M^{n}$, and $\nu$ the outward pointing conormal vector field to $M^{n}$ along $\partial M$. Then for $1 \leq k \leq n-1$ and for every closed conformal vector field $Y$ on $\bar{M}^{n+1}$, we have:

$$
\int_{\partial M^{n}}\left\langle T_{k}^{\infty} \nu, Y\right\rangle d s_{f}=\int_{M^{n}}\left\langle\operatorname{div}_{f} T_{k}^{\infty}, Y\right\rangle d v_{f}+c_{k} \int_{M^{n}} \phi H_{k, f} d v_{f}+c_{k-1} \int_{M^{n}} \phi\langle\nu, \nabla f\rangle H_{k-1, f} d v_{f} .
$$

If $\bar{M}^{n+1}$ has constant sectional curvature, we obtain by Corollary ??,

Proposition 3. Under the hypothesis of the proposition 2, if $\bar{M}^{n+1}$ has constant sectional curvature, then

$$
\int_{\partial M}\left\langle T_{k}^{\infty} \nu, Y\right\rangle d s_{f}=\int_{M^{n}}\left\langle\nabla f, T_{k}^{\infty} Y\right\rangle d v_{f}+c_{k} \int \phi H_{k, f} d v_{f}+c_{k-1} \int\left(\phi \mu_{1}+\frac{1}{n}\left\langle\nabla \mu_{1}, Y\right\rangle\right) H_{k-1, f} d v_{f}
$$

with $\mu_{1}=\langle\nabla f, \nu\rangle$.

Corollary 3. Under the hypothesis of the proposition 2., if $\bar{M}^{n+1}$ has constant sectional curvature and $f$ is constant then

$$
\int_{\partial M}\left\langle T_{k} \nu, Y\right\rangle d s_{f}=c_{k} \int_{M^{n}} \phi H_{k} d v_{f} .
$$

where $T_{k}$ is the classical Newton transformation.

If $f$ is constant, $H_{k}$ is a non zero constant and $Y$ is an homothetic vector field, we can assume that $\phi=1$, and get

$$
c_{k} H_{k} \operatorname{vol}\left(M^{n}\right)=\int_{\partial M}\left\langle T_{k}^{\infty} \nu, Y\right\rangle d s
$$

or equivalently

$$
\operatorname{vol}\left(M^{n}\right)=\frac{1}{c_{k} H_{k}} \int_{\partial M}\left\langle T_{k}^{\infty} \nu, Y\right\rangle d s .
$$

Proposition 4. Let $\varphi: M^{n} \longrightarrow \bar{M}^{n+1}$ be an immersed compact oriented hypersurface of boundary $\partial M$. Denoting by $N$ the global vector fields normal to $M^{n}$, and $\nu$ the outward pointing unit conormal vector field to $M^{n}$ along $\partial M$. Suppose that $\bar{M}^{n+1}$ is of constant sectional curvature, $f$ is constant and the $k^{\text {th }}$ mean curvature $H_{k}$ is non zero 
constant. Then for $1 \leq k \leq n-1$ and for every homothetic vector field $Y \in \bar{M}^{n+1}$ ), we have

$$
\operatorname{vol}\left(M^{n}\right)=\frac{1}{c_{k} H_{k}} \int_{\partial M}\left\langle T_{k}^{\infty} \nu, Y\right\rangle d s .
$$

Remark 2. An estimate of the integrant in formula 3.3 leads to an estimate of the volume of $M^{n}$ by the area of its boundary $\partial M$.

\section{REFERENCES}

[1] M. Abdelmalek, M. Benalili, K. Niedzialomski, Geometric Configuration of Riemannian submanifolds of arbitrary codimension, J. Geom 108 (2017), 803823.

[2] I. Agricola, M. Kraus, Manifolds with vectorial torsion. arXiv:1509.08944, [Math.DG].

[3] L. J. Alías, S. de Lira, J.M. Malacarne, Constant higher-order mean curvature hypersurfaces in Riemannian spaces. Journal of the Inst. of Math. Jussieu 5(4), 527-562 (2006).

[4] J. L. Barbosa, R. S'a Earp, Prescribed mean curvature hypersurfaces in $H^{n+1}(-1)$ with convex planar boundary I, Geometriae Dedicata 71 (1998), $61-74$.

[5] V. Bayle, Propriétés de concavité du profil isopérimétrique et applications. Thèse de Doctorat. Universitié Joseph-Fourier - Grenoble I, 2003.

[6] J. S. Case, A notion of the weighted for manifolds with $\sigma_{k}$-curvature density, arXiv: 1409.4455v2, [Math.DG].

[7] I. Corwin, Differential Geometry of Manifolds with Density, Rose-Hulman Undergraduate Mathematics Journal, Vol 7, Iss.1, Article 2 (2006).

[8] K. Castro, C. Rosales, Free boundary stable hypersurfaces in manifolds with density and rigidity results, arXiv: 1311.1952v1, November 2013.

[9] J. M. Espinar, Manifolds with density, applications and gradient Schrodinger operators, arXiv:1209.6162v6, Mars 2015.

[10] M. Gromov, Isoperimetry of waists and concentration of maps, Geom. Funct. Anal. 13 (2003), 178-215

[11] M. Koiso, Symmetry of hypersurfaces of constant mean curvature with symmetric boundary, Math. Z. 191 (1986), 567-574.

[12] R. Kusner, Global geometry of extremal surfaces in three-space. Doctoral Thesis, University of California (1985).

[13] F. Morgan, Manifolds with density. Notices Amer. Math. Soc. 52(8) :853-858 (2005).

[14] R. C. Reilly, Variational properties of functions of the mean curvature for hypersurfaces in space forms, J. Differential Geom. 8 (1973), 465-477.

[15] H. Rosenberg, Hypersurfaces of constant curvature in space forms, Bull. Sc. Math. 117 (1993), 211-239.

[16] G. Wei, W. Wylie, Comparaison geometry for the Bakry-Emery ricci tensor, j. differential geometry. 83 (2009) 377-405. 
(A. One) Ecole Supérieure de Management. Tlemcen Ageria.

E-mail address: abdelmalekmhd@yahoo.fr

Current address, A. Two: Dept. Mathematics, Faculty of Sciences BP 119 Université UABB Tlemcen. Algeria.

E-mail address: m_benalili@yahoo.fr 